\title{
Canadian Association of Neuropathologists Abstracts of papers and cases presented at the 42nd Annual Meeting
}

\author{
October 3-5, 2002 \\ Vancouver, British Columbia
}

Can. J. Neurol. Sci. 2002; 29: 394-401

The 42nd Annual Meeting of the Canadian Association of Neuropathologists was held from October 3-5, 2002 at the Granville Island Hotel in Vancouver, British Columbia. Local arrangements were co-ordinated by Dr. Ian Mackenzie.

The scientific sessions were comprised of 22 platform presentations and 17 diagnostic case presentations. These presentations were organized under the following headings: Tumors, Infectious Disorders, Pediatric and Developmental Neuropathology, Degenerative Diseases, Diseases of White Matter, and Miscellaneous Disorders. The Royal College of Physicians and Surgeons of Canada Lecturer was Dr. Alexander E. MacKenzie, Director of the Molecular Genetics Research Laboratory, Children's Hospital of Eastern Ontario and Professor of Pediatrics at the University of Ottawa. His lecture was entitled "Spinal Muscular Atrophy (SMA): ATale of Two Genes".

The meeting included a special symposium on multiple sclerosis. Included with this symposium was the Jerzy Olszewski Lecture presented by Dr. Donald W. Paty, Professor Emeritus, Division of Neurology at the University of British Columbia. Dr. Paty's presentation was entitled "Diagnosis and Management of Multiple Sclerosis from a MRI Perspective". Additional lecturers in this symposium were given by two distinguished members of the Canadian Association of Neuropathologists. Dr. G.R. Wayne Moore, Clinical Professor in the Department of Pathology and Laboratory Medicine at the University of British Columbia delivered a lecture entitled "Multiple Sclerosis: MRIPathology Correlations". The symposium was completed by a lecture by Dr. Samuel K. Ludwin, Professor in the Department of Pathology of Queen's University, entitled "Pathology and Pathogenesis in MS: Clinical and Experimental Studies".

\section{PLATFORM PRESENTATIONS}

\section{Focal myositis, report of three cases.}

\section{RAO (Downstate Medical Center and Kings County Hospital Center, Brooklyn, NY).}

Focal myositis, described by Heffner, Armbrustmacher, Earle (Cancer 1977;40:301-306) is a distinct clinicopathological entity. It is a benign localized myositic process affecting a single skeletal muscle. Clinically it presents as a mass often misdiagnosed as a "sarcoma". High rate of spontaneous regression and resolution, even with partial therapy, is a notable feature. Pathogenesis is not understood and speculated to be a pseudotumoral process in response to a subclinical injury. Three cases of focal myositis are presented. The patients were 28 (female), 60 (female), and 51 (male) years old; and clinically presented as neck, thigh, and chest wall masses respectively. The last patient was HIV and hepatitis C positive. Myopathic changes, prominent interstitial fibrosis with pattern similar to hepatic cirrhosis, and inflammatory cell infiltrates were seen.
The inflammatory cells were both $\mathrm{B}$ and T cells. No recurrence or evolution into polymyositis was noted. A single case of focal myositis is documented in a hepatitis $\mathrm{C}$ positive patient (Alnigenis, Clinical \& Experimental Rheumatology 17(5):631) and none in any HIV positive patients.

\section{Intracerebral hemorrhage associated with ephedrine usage.}

M.E. JENKINS, B. DEMAERSCHALK and L.C. ANG

(Departments of Pathology and Clinical Neurological Sciences, London Health Science Centre and UWO).

Intracerebral hemorrhage has been associated with the use of ephedrine and other sympathomimetic agents (phenylpropanolamine and pseudoephedrine) in over-the-counter medications. Some cases of hemorrhage have been linked to abuse of medications, but other cases are reported at recommended dosages. The mechanism underlying the hemorrhage is postulated to be the result of drug-induced CNS vasculitis or transient hypertensive episodes. There have been rare cases of CNS vasculitis based on biopsy findings, although the weight of 
the evidence has been angiographic. To our knowledge, no autopsy cases have been reported previously.

A previously healthy 49-year-old woman began taking a diet suppressant, Xenadrine (estimated 20-40 mg of ephedrine daily) five days prior to her presentation. She was not on any other medication, including anticoagulants. There was no history of hypertension, bleeding disorders, diabetes or heart disease, although she was a smoker. She developed a sudden onset of lightheadedness, vertigo and right temporal headache followed by dilated right pupil, left hemiplegia and progressive coma. MRI showed a large right thalamic hemorrhage. Four days later she was declared brain dead and ventilator support was withdrawn. The autopsy confirmed thalamic hemorrhage but no vasculitis was demonstrated after careful histological examination. We propose in our case, given the location of the hemorrhage and the lack of vascular pathology, the hemorrhage was the likely result of an ephedrine-induced hypertensive episode.

\section{The concept of gliomas as a "Travelling Wave": The application of a mathematical model to high- and low- grade gliomas.}

\section{K.R. SWANSON and E.C. ALVORD, JR. (University of Washington, Seattle WA).}

Although the histologic type and grade of a glioma can be documented by most neuropathologists, the rate of growth and degree of infiltration are generally only implied as rough averages or ranges based on past experiences and are never actually defined for any particular patient. We have developed a mathematical model of glioma growth based on data derived from currently available medical imaging techniques (anatomically accurate to $1 \mathrm{cu} \mathrm{mm}$ ). Recently we have studied one individual with an untreated glioblastoma and a series of patients with untreated low-grade gliomas, all with repeated scans. The model's prediction, that the "edge" of the detectable glioma should behave as a "travelling wave" and follow Fisher's approximation that the velocity equals twice the square root of the product of the growth rate and diffusion coefficient, has been confirmed. The implication is that the prognosis of any individual patient can be predicted if two sets of scans, separated in time for a significant increase in growth to be measured, can be obtained before treatment is begun. The effect of any subsequent treatment can then be determined by comparison of the actual and expected course in that patient. This should obviate the need for large numbers of patients with "similar" tumors, the homogeneity of the group being always suspect because of wide ranges in individual growth rates and degree of invasiveness.

\section{MAP2 immunohistochemistry in glial tumours.}

P. GOULD, L. CANTIN, C. PICARD, D. LACERTE, A. TURMEL (Hôpital de l'Enfant-Jésus du CHA, Quebec City, PQ, Canada).

The microtubule associated protein 2 (MAP-2) is normally found in neuronal dendrites but has also been identified in glial tumours, including oligodendrogliomas. In order to better understand the distribution of MAP-2 immunoreactivity in surgical neuropathology practice, a series of glial tumours operated at this institution between January 2001 and June 2002 were examined for MAP-2 immunoreactivity using a commercially available mouse monoclonal antibody known to be specific for the $2 a, 2 b$ and $2 c$ isoforms (Chemicon MAB364).

We confirmed that normal adult white matter shows virtually no staining for MAP-2. Although oligodendroglial tumours stain well for MAP-2, MAP-2 immunoreactivity was also seen in small, oligodendrocyte-like cells in both low-grade and highgrade astrocytomas as well as in most glioblastomas. MAP-2 staining was limited to rare isolated cells in cases of ependymoma with increased staining seen in anaplastic ependymomas. One DNET-associated lesion showed minimal staining of oligodendrocyte-like cells.

MAP-2 immunohistochemistry is useful to assess the cellular detail in primary brain tumours, but MAP-2 immunostaining cannot be recommended as a means to quantify the oligodendroglial component of a tumour.

\section{Oligodendrogliomas, a combined histologic and genotyping approach.}

M-C. GUIOT, M. LAFLEUR, R.LEBLANC*, R.F. DEL MAESTRO*, D. SIRHAN*, A. OLIVIER* and J.B. RICHARDSON (Departments of Neuropathology and *Neurology-Neurosurgery, Montreal Neurological Hospital, McGill University).

Allelic loss of chromosome $1 \mathrm{p}$ has been shown to predict survival and treatment response in oligodendrogliomas. For the past two years, we have applied to a subset of primary and recurrent oligodendrogliomas, grade II and anaplastic, a panel of markers including LOH for 1p and 19q, p53 mutations, EGFR amplification and deletion of CDKN2. All the tests were performed with DNA extracted from formalin fixed paraffinembedded tissue. These results were compiled with the goal of identifying subsets of tumors and establishing correlations with the histology and survival.

We selected 53 oligodendrogliomas, 27 grade II and 26 anaplastic tumors, in patients ( 22 women and 31 men), age 20 to 70 years (mean 46). Loss of heterozygosity using PCR technique was present in $34 \%$ of the cases for $1 p, 43 \%$ for $19 q$ and $27 \%$ for $1 \mathrm{p}$ and $19 \mathrm{q}$. P53 mutation was detected by SSCPin $26.5 \%$ of the tumors. The presence of loss of $1 \mathrm{p}$ and a simultaneous mutation for p53 was found only in one case. EGFR amplification using multiplex PCR was found in only four tumors. Long term survival could not be appreciated, survival less than two years in anaplastic oligodendrogliomas was associated with intact $1 \mathrm{p}$.

These preliminary observations may help in selecting appropriate molecular markers to run in a clinical setting.

\section{Surveillance system for Creutzfeldt-Jakob disease in Canada.}

A. GIULIVI (Health Canada and the Creutzfeldt-Jakob Disease Surveillance System).

Objective of CJD-SS:

(1) To gather information for all cases of CJD identified to the Surveillance System since 1998 in order to establish a database for research on CJD in Canada.

(2) Participate in international studies to determine the 
epidemiology of variant CJD and to be made aware of any cases occurring in Canada.

Methods: In April 1998, all Canadian physicians involved in the care of probable cases of CJD had been informed about our project and have been asked to notify the CJD-SS, at the toll free number provided, in order that the Surveillance System can ensure complete follow-up. This would involve ensuring the collection of a blood sample from the patient for genetic sequencing, organizing the performance of an autopsy at time of death and obtaining permission from the patient's family to conduct a medical records review as well as have a questionnaire completed.

Data and results obtained: As of December 2001 there have been 279 referrals to the Surveillance System; 121are confirmed cases of CJD by autopsy or biopsy reports. The incidence of CJD in Canada for 1999 and 2000 is 0.92 and 1.04 respectively. There have been 10 confirmed familial cases and NO cases of vCJD reported so far.

Conclusions: The Surveillance System, with the invaluable cooperation of both the physicians who care for patients with a diagnosis of CJD as well as their families, has been able to reflect the expected incidence of CJD of one person per million population. We have also been able to carry out further study on familial cases of CJD in Canada as well as contribute to the ongoing worldwide research on CJD and vCJD.

\section{HIV-1 gp120 upregulates iNOS in human CNS in vitro.}

K.A. WALSH, J. MEGYESI, J. WILSON and R.R. HAMMOND (Departments of Pathology and Clinical Neurological Sciences, London Health Sciences Centre, University of Western Ontario).

Human and murine CNS cultures exposed to recombinant HIV-1 envelope protein (gp120) exhibit a number of perturbations that resemble neuropathological features of HIV-1 associated dementia (HAD). Recent experiments have focussed on oxidative mechanisms of neuroglial injury in this model. Following exposure to gp120, primary mixed human forebrain cultures exhibit increased iNOS production as detected by immunohistochemistry. Double immunofluorescent analysis by confocal microscopy suggests that the bulk of this iNOS production is in astrocytes. In primary murine astrocyte cultures, iNOS is also upregulated by gp120. Furthermore, in primary astrocyte cultures from wild type and iNOS-knockout mice iNOS expression is restricted to the wild type cells. In addition, when cultures are preincubated with ascorbic acid, iNOS upregulation is muted. These results suggest that in response to HIV-1 gp120 exposure, cells of the CNS exhibit features of oxidative stress, including iNOS upregulation, and this can be attenuated by antioxidant supplementation. (R. Hammond is supported by a grant from the Ontario HIV Treatment Network).

\section{Neuropathology of cerebral malaria.}

K.L. SCHMIDT, H. LAU, H. HUYNH, V. WHITE, T. TAYLOR, K. DOROVINI-ZIS. (Department of Pathology, Vancouver Hospital and University of B.C., College of Osteopathic Medicine, Michigan State University and Malaria Project, Blantyre, Malawi).

Cerebral malaria $(\mathrm{CM})$ is characterized by severe neurologic dysfunction, seizures and reversible coma. The pathogenesis of neurological dysfunction is poorly understood.

We examined the brains of 40 Malawian children (seven months to nine years) who died from CM (25), severe malarial anemia (SMA-8), coma of other causes (COC-7), and four agematched controls. Sections of cerebral hemispheres, brainstem, cerebellum and spinal cord were stained with H+E, LFB-E and $\mathrm{mAb}$ to $\beta$-APP to assess myelin/axonal damage. Additional sections from 27 patients (CM-14, SMA-7, COC-6, controls-2) were stained for fibrinogen to assess the permeability of the blood brain barrier (BBB). Ring hemorrhages were present in 20/25 CM, 2/8 SMA and 1/6 COC patients and were most frequent in the white matter. Areas of demyelination not associated with ring hemorrhages were present in addition to perivascular myelin pallor. Evidence of axonal injury adjacent to ring hemorrhages and independent of vascular changes was present in 22/25 CM, 4/8 SMA and 1/4 COC patients. The integrity of the $\mathrm{BBB}$ was compromised in all $\mathrm{CM}$ and a minority of SMAand COC cases. Leaky vessels were noted both around apparently intact microvessels and in association with ring hemorrhages and were most prevalent in the white matter. These findings suggest that diffuse axonal damage and increased BBB permeability contribute to the pathogenesis of coma in pediatric CM. Supported by NIH R41154.

\section{Cryptococcus neoformans at Vancouver Hospital and Health Sciences Centre (VHHSC): Epidemiology, microbiology and histopathology.}

\section{L.M. HOANG*, D.L. ROSCOE*, P. DOYLE*, M. FYFE**, J. MAGUIRE* (*VHHSC and UBC Department of Pathology and Laboratory Medicine, and **BCCDC Epidemiology).}

Cryptococcus neoformans is well-known to cause disease in the immunocompromised hosts, with only sporadic reported cases in the immunocompetent in North America. In 2002, an outbreak due to a rare subspecies of C. neoformans (var. gatii) was identified on Vancouver Island (VI), British Columbia. This unusual outbreak affected at least 40 immunocompetent individuals since 1999, bringing cryptococcal infections to both medical and media attention. The object of this investigation was to review the epidemiological, clinical and pathological features of all cryptococcal infections in patients admitted to VHHSC. Microbiology and pathology databases were searched for cryptococcal infections from June 01, 1997 to present. Twentyfour cases were identified through cultures $(n=16)$ and histopathology $(n=8)$. Fifty-four percent $(13 / 24)$ had central nervous system involvement while $40 \%$ (10/24) had pulmonic involvement. Limited clinical data were available on one patient with cryptococcemia. Between 1997-2001, the incidence ranged from three to five cases/year. However, in 2002, nine cases were identified. Subspeciation of 16 isolates is underway, with seven 
cases so far identified as var. neoformans and one as var. gattii. This retrospective analysis identified a sudden increase in incidence of cryptococcal infections at VHHSC in 2002. Most cases from 1997 on, involve the central nervous system. The relationship between the observed increase in incidence of cryptococcal infections at VHHSC and the VI outbreak warrants further investigations.

\section{Anatomical-molecular correlates of central nervous system pathology in thanatophoric dwarfism.}

P. SHANNON* S. KEATING** (Departments of Pathology and Laboratory Medicine, Toronto Western Hospital* and Mount Sinai Hospital ** and the University of Toronto, Toronto, Ontario).

Thanatophoric dwarfism is a lethal skeletal dysplasia resulting from an activating mutation in the fibroblast growth factor receptor 3 gene (FGFR3). Temporal lobe malformations are well-described, but the pathogenesis of the cerebral malformation is poorly understood.

We examined four cases of thanatophoric dysplasia seen at fetal autopsy. The infants ranged from 22-27 weeks gestational age. The brains showed an expanded temporal lobe with radially directed sulci and abnormal hippocampal formations. The germinal matrix in these brains is expanded over that of agematched controls, and there is as well an increase in subcortical labeling with MIB-1.

In thanatophoric dwarfism there is evidence of increased proliferative activity and cellular activation in the medial temporal lobe consistent with a mechanism implicating the activated FGFR3 gene. We suggest that the abnormal sulcation of the temporal lobes may be necessitated by an abnormally rapid hemispheric expansion, driven by a constitutively activated mitotic stimulus.

\section{Rhombencephalosynapsis as an incidental finding in a 50-year-old man.}

\section{S.A. KRAWITZ*, L.E. BECKER** and R.R. HAMMOND* ( $*$ London Health Sciences Centre, University of Western Ontario and **The Hospital for Sick Children, University of Toronto).}

We report the occurrence of rhombencephalosynapsis (RES) as an incidental finding at autopsy in a 50-year-old male, the oldest reported case to date. The deceased had chronic problems with alcohol dependency but no other significant medical or neurological history.

He was of average intelligence having graduated from high school and completed some college-level courses. RES is usually associated with other significant CNS and systemic malformations, often leading to intellectual impairment and death in infancy or childhood. The present case demonstrates that isolated RES may be clinically silent, pathologically discreet and likely compatible with a normal intellect and life expectancy.

\section{Familial Ohtahara syndrome.}

Y. ROBITAILLE, A. LORTIE, J. MICHAUD (Department of

Pathology and Medical Genetics, Ste-Justine Hospital, Montreal, QC).

Ohtahara syndrome is a rare, usually sporadic, infantile epileptic encephalopathy characterized by suppression bursts on EEG associated with olivary-dentate dysplasia.

We report the first instance of a familial occurrence of this syndrome. The mother, aged 28 years at the birth of the second sibling, was G2, P1, A0, V0. There had been onset of polyhydramnios at 27 weeks. Since then, she felt that short bouts of saccadic movements in utero resembled seizures. A cordocentesis yielded a normal karyotype. MRI at 32 weeks disclosed widespread cortical development delay of about two weeks duration with dilatation of the sub-arachnoid space. Delivery by $\mathrm{C}$-section was done at 33 weeks. APGAR scores were 5-8-8 and death ensued within two days in severe respiratory distress. There was generalized hypotonia associated with a palatine fissure and arthrogryposis as well as other more minor dysmorphic features. The first sibling had died two days after a 29 week C-section for loss of fetal movements and neonatal onset of seizures. An autopsy of the second sibling confirmed the presence of severe olivary-dentate dysplasia, compared with four age-matched control brain stems and previously reported cases. Putative pathophysiologic mechanisms will be discussed.

\section{Neuropathological features of glutaric acidemia type 1 (GA1) in an aboriginal cohort.}

C. FUNK, M.R. DEL BIGIO, and A. PRASAD (Department of Pathology and Section of Pediatric Neurosciences, Department of Pediatrics, University of Manitoba).

GA1 is an autosomal recessive disorder caused by deficiency of glutaryl-CoA dehydrogenase. This leads to accumulation of neurotoxic metabolites and excretion of large amounts of glutaric acid, 3-hydroxyglutaric acid, and glutaconic acid. A single homozygous mutation in the enzyme (GCDH IVS- $1+5 \mathrm{~g} \rightarrow \mathrm{t}$ ) has been identified in the aboriginal population from Manitoba/ Northwestern Ontario. In infancy, affected individuals experience sudden onset of encephalopathy and seizures, usually triggered by intercurrent illness. Residual deficits in survivors include generalized dystonia-choreoathetosis. Fifteen cases have been described.

We describe the neuropathological features of six additional cases from a homogenous genetic background (ages 8, 12, 16, 18 months, 7, 42 years; five male, one female). One case was megalencephalic, while two others had brains at the upper end of the normal size range.

All had severe neuron loss involving the caudate and striatum. The nucleus accumbens was less affected. Microglial activation was evident in subacute cases. All had reactive astrocytes. Other brain regions were unaffected with the exception of mild gliosis in the inferior olivary nucleus of the oldest patient. We are undertaking studies to determine the vulnerability of different neuronal populations in this disorder. [Funded by the Garrod Association and Manitoba Medical Service Foundation. We acknowledge the support of the GA 1 study group.] 


\section{Magnesium sulfate therapy is of mild benefit to young rats with kaolin-induced hydrocephalus.}

O. KHAN, T. ENNO, and M.R. DEL BIGIO (Department of Pathology, University of Manitoba).

Hydrocephalus causes damage to periventricular white matter at least in part through chronic ischemia. Magnesium sulfate, a calcium antagonist, has been shown to be protective in various models of neurological injury. We hypothesized that this agent would ameliorate the effects of experimental childhood-onset hydrocephalus. Hydrocephalus was induced in three- and fourweek-old rats by injection of kaolin into the cisterna magna. Tests of cognitive and motor function were performed on a weekly basis. In a blinded and randomized manner, magnesium sulfate was administered in two separate experiments once or twice daily (subcutaneous injection $0.17 / 100 \mathrm{~g}$ body weight), supplemented by osmotic minipump infusion $(3.7 \mathrm{mg} /$ day $)$ for two weeks, beginning two weeks after induction of hydrocephalus. The brains were then subjected to histopathological and biochemical analyses. At a starting dose of $23 \mathrm{mg} / \mathrm{kg} /$ day, we observed statistically significant improvement in performance on the roller test and evidence of reduced astroglial reaction. A higher dose was associated with sedation.

We conclude that magnesium sulfate might be mildly protective in hydrocephalus and we suggest that low serum magnesium levels should be avoided in infants with symptomatic hydrocephalus.

[Funded by a grant from the Canadian Institutes of Health Research. Mr. Khan holds a summer studentship award from the Children's Hospital Foundation.]

\section{Malignant transformation of an intraaxial supratentorial neurenteric cyst.}

\section{DUNHAM*, B. CURRY*, M. HAMILTON** (Foothills Medical Centre, University of Calgary, Departments of \\ *Neuropathology and **Neurosurgery).}

Neurenteric cysts (NCs) are rare benign developmental abnormalities usually arising at the anterior cervical-thoracic spinal cord as an extramedullary-intradural mass. Closely related to colloid cysts, NCs are thought to be derived from primitive endoderm, arising as a result of faulty separation of this endoderm from the notochord at three weeks of embryogenesis. NCs are cystic lesions, lined by a simple cuboidal-columnar epithelium that may demonstrate cilia and goblet cells. Management is typically surgical, with rare recurrences usually related to subtotal resection.

Malignant transformation of the epithelial component of NCs has been reported twice, both in extraaxial locations \{Ho et al [Neurosurgery 1998;43(6);1474-1477] and Sahara et al [Journal of Neurosurgery 2001;95;341-345]\}. We describe a 58-year-old female with a well-differentiated papillary adenocarcinoma arising intraaxially in the right parietal region. No other primary tumor has been identified in our patient after seven months of follow-up.

To our knowledge, this would be the first reported case demonstrating malignant transformation of a $\mathrm{NC}$ in a supratentorial-intraaxial location.

\section{Myxoid/round cell liposarcoma can occur in the paraspinal area.}

\section{S.L. WHITE, B.H. LIWNICZ* (*Loma Linda University} Medical Center, Loma Linda, California).

A 57-year-old male with history of multiple liposarcomas presented with pain radiating from his back to his right leg, right lower extremity weakness, decreased sensation on his right foot, and bowel incontinence. MRI showed a tumor in the right posterior paraspinal soft tissue with intraspinal extension and compression and displacement of the thecal sac from L5-S2.

It was removed and submitted to pathology. The tumor was composed of areas with variable cellularity. The majority of the tumor was composed of small round cells with occasional adipocytes. The remaining tumor was less cellular with a myxoid matrix containing small spindle cells and small lipoblasts. To our knowledge, myxoid/round cell liposarcoma of the paraspinal area is rare. In other sites, myxoid/round cell liposarcoma is fairly common and occurs most frequently in the lower extremity thigh and popliteal region. Myxoid/round cell liposarcomas behave more aggressively with increased round cell component, regardless of location. They tend to metastasize to other soft tissue locations such as the opposite extremity and retroperitoneum. Follow-up MRIs of the reported case showed multiple lesions consistent with myxoid/round cell liposarcoma, throughout the pleura, neck, back and retroperitoneum. Genetically, myxoid/round cell liposarcomas are characterized by a reciprocal translocation between chromosomes 12 and 16 resulting in the CHOP-FLS gene fusion. Myxoid/round cell liposarcomas, including this one, are treated by resection followed by radiation and chemotherapy.

\section{Quantitative analysis of APPand $\beta$-amyloid in nondementia cases, referring to diseased and non- diseased brains, as well as aging.}

S. HASHIMOTO*, R. ITO**, Y. HAYASHI**, T. ITOH**, and T. SATOU** (*Department of Pathology, PL Hospital, **Second Department of Pathology, Kinki University School of Medicine).

In autopsies of nondementia cases, with a comparison of diseased and nondiseased brains, analyses of amyloid precursor protein (APP) and $\beta$-amyloid (BA) were performed in relation to age. $50 \mu \mathrm{m}$ vibratome sections were immunohistochemically stained with BA and usual paraffin sections were stained with APP. Positive ganglion cells and dots of APP and BA, which appeared in the neuropil, were histometrically analyzed in the superior frontal gyrus, including the motor area, and parahippocampal gyrus in 72 autopsies of nondementia brains.

The specimens consisted of 33 diseased and 39 nondiseased brains, with an age distribution from 0 (six hours after birth) to 92 years old. In the diseased brains, APP positive nerve cells were significantly increased with age from 40 years to approximately 60 to 70 years old, while BA was continuously and significantly increased up to the age of 90 . Increases of APP dots were not significant. In nondiseased brains, APP and BA increased with age; this, however, was not statistically significant. In nondementia cases, it is possible that multiple factors of the disease process in the central nervous system have 
a significant effect on the increases of APPand BAwith age. An analysis of these factors should be performed in the future.

\section{Dissecting the functional and pathological significance of neuronal intranuclear rodlets.}

J. WOULFE*, R. HAMMOND**, D. GRAY*\#,W. PRICHETT* and D.G. MUNOZ" (The University of Ottawa, The Ottawa Hospital, and The Ottawa Health Research Institute*, The University of Western Ontario and The Lawson Research Institute**, The Ottawa Regional Cancer Centre ${ }^{\sharp}$, Banco de Tejidos para Investigacion Neurologica, Madrid, Spain ${ }^{\text {II). }}$.

There is increasing appreciation of a structural basis for functional compartmentalization of neuronal nuclei. Intranuclear bodies including coiled bodies, nucleoli, and interchromatin granules play an important role in this. We have previously described the existence of unique intranuclear inclusions (INRs) in neurons of the normal human brain which exhibit a widespread but anatomically selective topographic distribution. These inclusions are intensely immunoreactive for the neuronspecific cytoskeletal protein class III beta tubulin.

Their functional and pathological relevance is uncertain. However, we previously demonstrated that they are markedly reduced in the brains of patients with Alzheimer's disease (AD) relative to those with dementia with Lewy bodies and normal controls. In the present study, we confirm and extend these findings; comparing INR densities in AD brain versus those in a variety of other neurodegenerative diseases. In addition, we demonstrate immunoreactivity of INRs for glucocorticoid receptor (GR); a finding which may implicate these structures in the mechanisms underlying intranuclear steroid signalling. Finally, the presence of INRs immunoreactive for tubulin and GR is documented in mouse brain at both the light and electron microscopic levels, thereby providing a valuable tool to further study the functional and neuropathological significance of these enigmatic inclusion bodies.

\section{The neuropathology of spinocerebellar ataxia type 17 (SCA-17).}

\section{A.H. KOEPPEN, A.C. DICKSON and O. RIESS (VA Medical Center, Albany, New York, USA; and University of Tübingen, Germany).}

Most of the autosomal dominant ataxias are caused by cytosine-adenine-guanine (CAG) expansions in coding regions of the mutated genes. The TATA-binding protein (TBP) gene on chromosome 6q27 was a candidate for a polyglutamine (polyQ)related dominant ataxia because it contains a CAG repeat. A monoclonal antibody originally raised against TBP ("1C2"; Lescure et al, EMBO J 1994;13:1166-1175) recognizes polyQ and is now widely used in established or putative polyQ diseases. Pathological CAG expansion of the TBP gene was recently reported from several countries, and the disease was designated SCA-17. This mutation was identified in a previously published German family with dominant ataxia (Fig. 2 in Koeppen et al, Arch Neurol 1981;38:158-164). Clinical findings included progressive ataxia, dystonia, and cognitive decline. Autopsies were performed on three sisters. The brains weighed $1,125 \mathrm{~g}$, $1,100 \mathrm{~g}$, and $903 \mathrm{~g}$, respectively. Routine histology revealed "pure" cerebello-olivary atrophy. However, monoclonal antibodies against TBP (including 1C2) showed pan-nuclear reaction product in the neurons of the basis pontis, the nucleus raphae, the midbrain tegmentum, the stellate and basket cells of the cerebellar cortex, the dentate nucleus, the nucleus basalis of Meynert, the dorsomedian nucleus of the thalamus, and the pyramidal cells of the cerebral cortex. Only a few nucleoliform inclusions were present. They reacted with anti-TBP and antiubiquitin. However, cells with pan-nuclear TBPreaction product were ubiquitin-negative.

\section{Primary lateral sclerosis with ubiquitinated inclusions in lower motor neurons.}

\section{A.W. CLARK, C. WHITE (University of Calgary, Dept of Pathology and Laboratory Medicine and Dept of Clinical Neurosciences).}

Remarkably few autopsy studies of primary lateral sclerosis (PLS) have been published. As with clinical studies, autopsies have revealed little evidence of lower motor neuron involvement. Ubiquitinated inclusions in lower motor neurons are known to be a common finding in amyotrophic lateral sclerosis. We recently conducted a postmortem neuropathologic evaluation of brain and spinal cord from a 73-year-old woman with onset of PLS in July 1998 and death in February 2002. Dragging of the right foot and leg was followed by a progressive and severe spasticity, with only moderate weakness, largely confined to the legs. Electromyography of leg muscles revealed no fibrillations, positive sharp waves, or fasciculation potentials. An MRI ruled out cord compression. The clinical findings met proposed criteria for PLS (Pringle et al 1992). Postmortem neuropathologic exam revealed extreme gliosis and atrophy or loss of Betz cells in parts of the motor cortex near the interhemisphere fissure, bilaterally. The corticospinal tracts were severely degenerated in the spinal cord, more subtly affected at progressively rostral levels. The spinal anterior horns showed gliosis. Ubiquitin immunostains revealed frequent cytoplasmic inclusions of anterior horn cells in the lumbar spinal cord. Ubiquitin immunostaining may detect involvement not otherwise apparent from clinical or pathologic assessment, and should be conducted on all autopsy cases of PLS.

\section{Leukoencephalopathy with neuroaxonal spheroids: Report of a Canadian case.}

G. MEDVEDEV*, G.R.W. MOORE*, D.L. WEBBER**, T.P. SELAND**, M. ROYTTA ${ }^{+}$, K. SUZUKI ${ }^{\text {Il }}$ (*Vancouver General Hospital and University of British Columbia, Vancouver, BC; **Kelowna General Hospital, Kelowna, BC; ${ }^{+}$University of Turku, Turku, Finland; "University of North Carolina, Chapel Hill, NC, USA).

Leukoencephalopathy with neuroaxonal spheroids is a very rare autosomal dominant disorder. As far as we are aware this is the first reported case in Canada. The patient was a 41-year-old male who presented with three-year progressive history of ataxia, spasticity, dementia and obtundation. The patient's father, who died at an age of 40 years, had a neurologic illness, the details of which are not available but required his institutionalization. A paternal cousin is said to have "multiple 
sclerosis". Postmortem MRI showed further progression of the white matter abnormalities noted during life, with relative sparing of the subcortical U-fibres. Grossly, the formalin fixed brain showed fronto-temporal-parietal atrophy and ill-defined areas of gray discolouration within the hemispheric white matter, particularly periventricularly.

Light microscopy showed vacuolated regions of myelin loss, large axonal swellings and small numbers of macrophages containing sudanophilic material distributed widely throughout the hemispheric white matter with subcortical U-fibre sparing. Vacuolar degeneration of the corticospinal tracts was noted in the brainstem and spinal cord. The findings are characteristic of this unusual white matter disorder.

(Supported by the Multiple Sclerosis Society of Canada and Berlex).

\section{Axon loss in the corticospinal tracts in multiple sclerosis.}

G.C. DELUCA, G.C. EBERS, M.M. ESIRI (University Department of Clinical Neurology, University of Oxford, Oxford, $U K)$.

Pathological studies in multiple sclerosis (MS) have classically emphasized the relative preservation of axons in comparison to myelin. Recent evidence, however, demonstrates that axon loss is also significant, affects long tracts such as the corticospinal tracts and relates closely to functional disability. Accordingly, the cause of this axonal loss is the focus of the current investigation.

Postmortem material from the cerebrum (internal capsule), brainstem (midbrain, mid-pons and mid-medulla) and several levels of the spinal cord (high and low cervical, high and low thoracic and lumbar) of 55 MS patients and 32 matched controls have been selected to analyse the lateral corticospinal tracts quantitatively via a semi-automated computational procedure. Myelin and axon-stained sections have been prepared to estimate the notional area and axon density of the lateral corticospinal tracts respectively. Our preliminary results indicate that there is a significant reduction of the area and axon density of the lateral corticospinal tracts at all levels of the spinal cord in MS patients when compared to controls. Of the fibres lost in MS, we have found that small fibres $(<3 \mu \mathrm{m})$ seem to be particularly affected with large fibres remaining relatively preserved. By estimating the plaque load in the cerebrum, brainstem and the spinal cord, we hope to advance our understanding of the role of demyelination in axon loss seen in MS.

[Funded by the Canadian MS Society.] 


\section{Titles of Diagnostic Case Presentations}

1. Erdheim-Chester disease of the central nervous system

J.M. BILBAO and J. LAU (Department of Pathology, St.

Michael's Hospital, University of Toronto)

2. Hyaline body myopathy

WM. HALLIDAY, P. SHANNON, M. RAFAY and V. BRIL (Division of Neuropathology, Toronto Western Hospital at the University Health Network and University of Toronto)

\section{Cerebral amyloidoma}

C. HAWKINS", B. GRAY*, R. MOULTON ${ }^{\phi}$ and J.M. BILBAO ${ }^{\#}$ (Departments of Pathology , Radiology* and Neurosurgery ${ }^{\star}$, St. Michael's Hospital, Toronto)

4. Cerebral amyloid angiopathy (A $\beta$ peptide) with multifocal perivascular lymphocytic infiltrates and focal mural lymphocytic infiltrates.

J.P. ROSSITER, M. MELANSON* (Divisions of

Neuropathology and *Neurology, Kingston General Hospital and Queen's University, Kingston, Ontario)

\section{Pigmented villonodular synovitis}

E. JOHNSON (Department of Laboratory Medicine and Pathology, University of Alberta)

\section{Malignant intracerebral rhabdoid meningioma}

B. LACH, A.M. ALKHANI (Department of Pathology and Laboratory Medicine, Department of Neurosciences, King Faisal Specialist Hospital \& Research Centre, Riyadh, Kingdom of Saudi Arabia)

7. Composite pleomorphic xanthoastrocytoma and ganglioglioma

B. CURRY and T.S. MYLES (Department of Pathology [Neuropathology] and Clinical Neurosciences, University of Calgary at Foothills Medical Center)

\section{Primary myofibroblastoma of dura mater}

B. LACH, M. HASSOUNAH (Department of Pathology and Laboratory Medicine, Department of Neurosciences. King Faisal Specialist Hospital and Research Centre. Riyadh, Kingdom of Saudi Arabia)

\section{Dentato-olivary dysplasia}

D.H. GEORGE (Alberta Children's Hospital and Calgary Laboratory Services, Calgary)

10. Malignant melanotic neuroectodermal tumor of infancy

B. LACH, M. OTIBI, E. AL SHAIL, M.A. DABABO

(Department of Pathology and Laboratory Medicine, Department of Neurosciences, King Faisal Specialist Hospital and Research Centre, Riyadh, Kingdom of Saudi Arabia)

11. Neuroglial choristoma of neck

L. RESCH (Department of Pathology and Laboratory Medicine, IWK Health Centre)

12. Schwannoma with giant "neuroblastomatous" rosettes

I.R.A. MACKENZIE (Vancouver General Hospital and University of British Columbia)

\section{Gastrointestinal stromal tumor with skenoid fibers}

P. SHANNON (Department of Pathology and Laboratory Medicine, The Toronto Western Hospital and The University of Toronto)

14. Neuronal intranuclear hyaline inclusion disease

L. RESCH (Department of Pathology and Laboratory Medicine, IWK Health Centre)

15. Multisystem tract degeneration, query pernicious anemia as etiology

E.C. ALVORD, JR. (Neuropathology, University of Washington Medical School)

16. Cree encephalitis, with marked CSF increase of $\alpha$ interferon

Y. ROBITAILLE (Department of Pathology \& Cell Biology, University of Montreal, Ste-Justine Hospital, Montreal, Quebec)

17. Posterior leukoencephalopathy with thrombotic thrombocytopenia-related thrombotic microangiopathy

A.S. EASTON (Department of Laboratory Medicine and Pathology, University of Alberta, Edmonton) 